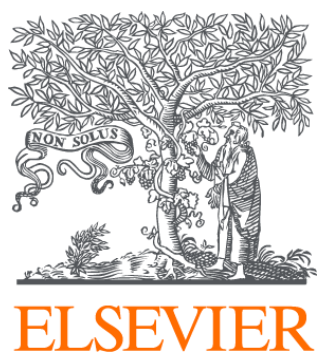

Since January 2020 Elsevier has created a COVID-19 resource centre with free information in English and Mandarin on the novel coronavirus COVID-

19. The COVID-19 resource centre is hosted on Elsevier Connect, the company's public news and information website.

Elsevier hereby grants permission to make all its COVID-19-related research that is available on the COVID-19 resource centre - including this research content - immediately available in PubMed Central and other publicly funded repositories, such as the WHO COVID database with rights for unrestricted research re-use and analyses in any form or by any means with acknowledgement of the original source. These permissions are granted for free by Elsevier for as long as the COVID-19 resource centre remains active. 
Short Communication

\title{
Parental perceptions of COVID-19-like illness in their children
}

\author{
A. Hodson ${ }^{\text {a, }}{ }^{*}$, L. Woodland ${ }^{\text {b }}$, L.E. Smith ${ }^{\text {b }}$, G.J. Rubin ${ }^{\text {b }}$ \\ ${ }^{a}$ King's College London, Department of War Studies, Strand Campus, London, WC2R 2LS, UK \\ ${ }^{\mathrm{b}}$ King's College London, Institute of Psychiatry, Psychology and Neuroscience, Department of Psychological Medicine, Weston Education Centre, Cutcombe \\ Road, London, SE5 9RJ, UK
}

\section{A R T I C L E I N F O}

Article history:

Received 26 October 2020

Received in revised form

22 January 2021

Accepted 14 February 2021

Available online 2 March 2021

\section{Keywords:}

Symptom attribution

Cough

Fever

Symptom perception

Isolation

\begin{abstract}
A B S T R A C T
Objectives: The objective of the study is to explore parents' perceptions of COVID-19-like symptoms in their child and attitudes towards isolating from others in the household when unwell.

Study design: The study used qualitative, semistructured interviews.

Methods: The study involved thirty semistructured telephone interviews with parents of children between 4 and 18 years. Thirty semistructured telephone interviews with parents of children between 4 and 18 years. Results: We found four themes relating to symptom attribution ('normalising symptoms', 'err on the side of caution', 'experience of temperature', 'symptoms not normal for us'). In general, parents were more likely to attribute symptoms to COVID-19 if a temperature was present or the symptoms were perceived as 'unusual' for their family. Four themes relating to self-isolation ('difficult to prevent contact with children', 'isolation would be no different to lockdown life', 'ability to get food and supplies', 'limited space'). Parents believed they would find isolation within the household difficult or impossible if they had dependent children, had limited space or could not shop for groceries.

Conclusions: The findings highlight complexities in symptom perception, attribution and household isolation. We suggest that they can be overcome by (a) providing better guidance on what symptoms require action, (b) providing guidance as to how to prevent infection within the household and (c) by supporting families with grocery shopping through a potential second or third wave.

(c) 2021 The Authors. Published by Elsevier Ltd on behalf of The Royal Society for Public Health. This is an open access article under the CC BY license (http://creativecommons.org/licenses/by/4.0/).
\end{abstract}

Children can spread certain illnesses readily at school and, in order to prevent outbreaks, are encouraged to stay at home when ill or until they are no longer infectious. ${ }^{1}$ How thoroughly children and parents adhere to the rules around sickness absence is unclear. In England, schools were closed nationally from 23rd March 2020, to prevent the spread of COVID- $19^{2}$ and reopened to all children in September. There have been concerns that some parents may continue to send children to school when experiencing the symptoms of COVID-19, which include fever and cough. ${ }^{3}$

Sending a child to school while symptomatic, rather than keeping them at home and arranging a test for COVID-19 as recommended by national guidance, may depend partly on how parents interpret their child's symptoms ${ }^{4}$ and partly on whether the parent is willing and able to keep the child off school. This in turn may require the parent to take time off work and affect the child's education. However, little is known about factors that are at play in this decision-making process.

\footnotetext{
* Corresponding author.

E-mail address: ava.hodson@kcl.ac.uk (A. Hodson).
}

In this article, we use data from interviews that explored parents' perceptions of COVID-19-like symptoms in their child and attitudes towards isolating from others in the household when unwell.

Two interviewers conducted semistructured telephone interviews lasting approximately $75 \mathrm{~min}$ between 15th and 21st April $2020(n=30$, female $=20)$. All participants were aged 18 years or older and were the primary caregiver to a child who, from March 23rd, 2020 was not attending preschool or school in England because of COVID-19. At the time of data collection, the recognised symptoms of COVID-19 were a new, continuous cough and fever. Loss of sense of smell or taste was added on May 18th, $2020{ }^{5}$ At the time of interviewing, guidance stated that individuals who suspected they had symptoms should isolate as best they could from others in the household. ${ }^{6}$

A broad discussion guide was used, covering psychological wellbeing, educational activity while at home and adherence to social distancing guidance. Results for these aspects of the interviews will be reported elsewhere. In this manuscript, we focus on responses relating to symptom perception, attribution, and how parents thought they would react to the presence of symptoms among their 
Table 1

Supporting quotes for identified themes.

Topic
Theme

Quote (Participant number; Age; Gender; Ethnicity; Highest education level; Education level of oldest child living at home, Number of children)

Symptom attribution

Normalising symptoms

Err on the side of caution

Experience of temperature

Symptoms not normal for us

Ability to isolate

Difficult to prevent contact with children

Isolation would be no different to lockdown life
'I've not done night shift work before, there's times where I've felt a bit odd, and I've thought ...' 'Are these symptoms?' 'Am I showing symptoms of corona?' But then you think, 'Actually, no I'm just tired,' and, 'Actually, I've got a bit of a sore throat, because-' (P27; 25-49; Male; White; Higher education or equivalent; Secondary, 3)

'My five-year-old did have cough, but then he gets cough around that time anyway ... cough is one of those things, you know if you get dust in the house, if it gets dusty or hay fever or whatever' (P22; 25-49; Female; BAME; A-Levels or equivalent; Primary; 5) 'A cough ... it only lasted a day ... she didn't have any other symptoms [and] she has suffered with a cough since she was young' (P2; 25-49; Female; White; A-level or equivalent; Preschool, 2)

'It was ... 24, $48 \mathrm{~h}$ of this horrible tickly cough that wouldn't go away but it wasn't continual and there was no other symptoms. Neither of the kids picked it up. So, I can only assume it was something more likely linked to hay fever or a cold' (P26; 25-49; Female; White; Higher education or equivalent; Primary; 2)

'You can carry [COVID-19], but you don't have it yourself ... I just wouldn't wanna be responsible for anyone's illness' (P13; 25-49; Female; BAME; GCSEs or equivalent; Primary, 1)

'If we thought we had any symptoms we'd be protecting ourselves' (P24; 25-49; Female; White; Higher education or equivalent; Primary, 1)

'As soon as they get a cold or a cough, you're thinking, 'Is this the coronavirus?' And I know that's a bit paranoid, but...' (P15; 25-49; Male; White; Higher education or equivalent; Secondary, 4)

'definitely have to stay in if they were showing symptoms' (P4; 25-49; Female; BAME; Higher education; Secondary, 2)

'I'll just keep her in to be safe ... and err on the side of caution for once.' (P5; 25-49; Female; White; A-level or equivalent;

Secondary, 2)

'I was definitely self-isolating ... because I didn't want to pass it to my nana and because I know that that's the best thing to do, with any virus. Even regardless of coronavirus' (P11; 25-49; Female; BAME; Higher education or equivalent; Secondary, 1) 'Before I was a oh-just-suck-it-up, he'll-be-fine, kind of mum. Unless there was a temperature' (P11; 25-49; Female; BAME; Higher education or equivalent; Secondary, 1)

'Well if she didn't have a temperature, she had a cough ... or a bit of a cold, then I'd send her in, yeah' (P1; 25-49; Female; BAME; Higher education or equivalent; Secondary, 1)

'Of course, we're concerned, because obviously I had temperature' (P14; 25-49; Male; BAME; Higher education or equivalent;

Secondary, 4)

'He got a temperature for about $24 \mathrm{~h}$. I had a temperature, a sore throat and a headache for about two days. Possibly, we could have had mild symptoms. But I don't know, without being tested we won't know' (P23; 25-49; Female; White; British; Higher education or equivalent; Primary, 2)

'We know each other, we'll know if something gets to that stage, we would go and get tested' (P10; 25-49; Male; White; Higher education or equivalent; Secondary, 2)

'Everyone was so tired. I mean so tired, they couldn't watch telly, they couldn't do anything ... that was what was weird' (P8; 25-49; Female; White; Higher education or equivalent; Secondary, 4)

'For [them] to lose [their] appetite is a key indicator if something is wrong [sic]' (P11; 25-49; Female; BAME; Higher education or equivalent; Secondary, 1)

'I don't know how that would work' (P4; 25-49; Female; BAME; Higher education; Secondary, 2)

'That would break my heart, if I couldn't go near him. I genuinely don't know how I'd do that' (P12; 25-49; Female; White; A-Level or equivalent; Primary, 1)

'Kids don't know boundaries ... it would be impossible for me and my husband not to get it I think' (P7; 25-49; Female; White; ALevel or equivalent; Preschool, 1)

'Unless [we] were ... wearing gloves and a mask 24/7 to look after him [they could avoid catching COVID-19] ... But even then, I think ... we'd get it, because it's just too difficult with a three-year-old' (P7; 25-49; Female; White; A-Level or equivalent; Preschool, 1) 'If it was me or [my partner], I don't think it would be much of a problem. We could just shut ourselves away, but if the kids got it, that would be different, and I think we'd almost certainly catch it from them' (P8; 25-49; Female; White; Higher education or equivalent; Secondary, 4)

'I think we just accept the fact that we wouldn't be able to do that ... if one goes down, probably all of us would go down' (P9; 18-24; Female; White; GCSEs or equivalent; Primary, 2)

'I have been panicking, which is why I've been staying in and extra precautions, because I feel if I was to get ill, I don't know how he'd cope looking after me and the kids' (P30; 25-49; Female; White; GCSEs or equivalent; Secondary, 3)

'It would be basically impossible to. Especially my one-year-old and two-year-old. The one-year-old, the two-year-old, the fouryear-old, they're constantly wanting to be with me ... And he's breastfeeding as well' (P22; 25-49; Female; BAME; A-Levels or equivalent; Primary, 5)

'My little girl, she wouldn't understand it at all. It's no good having the conversation with her 'cause she just won't understand it' (P10; 25-49; Male; White; Higher education or equivalent; Secondary, 2)

'They wouldn't understand 'no, you can't go and see mummy' or 'no you can't go and talk to daddy'. I think that would be quite hard' (P9; 18-24; Female; White; GCSEs or equivalent; Primary, 2)

'That's a real worry for me because if I was ill, it's just me and the children, that is a real worry' (P29; 50-65; Female; White; Higher education or equivalent; Secondary, 2)

'Being ill on my own with the kids ... was a bit of a pest. So, it was Mr YouTube had to look after them' (P23; 25-49; Female; White; British; Higher education or equivalent; Primary, 2)

'It would be hard for anyone to look after a kid or kids on their own whilst they're also trying to look after [others in the household]' (P7; 25-49; Female; White; A-Level or equivalent; Preschool, 1)

'Well apart from going to the park or ... shopping, very similar ... So yeah, I'd adapt to it. Plus I'd adapt to it even more because it's your life' (P28; 50-65; Male; White; A-Levels or equivalent; Secondary, 1)

'We're not going anywhere; it's going to be very difficult for them to catch anything. We're just at home' (P18; 25-49; Female; White; Higher education or equivalent; Primary, 6)

'They're that much self-isolated anyway' (P6; 25-49; Female; BAME; Higher education; Secondary, 2)

'It would be easy, we've done it for six weeks. Another 14 days wouldn't do anything, would it?' (P30; 25-49; Female; White; GCSEs

or equivalent; Secondary, 3 ) 
Table 1 (continued)

\begin{tabular}{|c|c|}
\hline \multicolumn{2}{|l|}{ Topic } \\
\hline Theme & $\begin{array}{l}\text { Quote (Participant number; Age; Gender; Ethnicity; Highest education level; Education level of oldest child living at home, } \\
\text { Number of children) }\end{array}$ \\
\hline Ability to get food and supplies & $\begin{array}{l}\text { 'There's big network in my town, so if anyone is having to self-isolate, you can contact the [local] Coronavirus Project' (P19; 25-49; } \\
\text { Female; Separated; White British; A-Levels or equivalent; Secondary, 5) } \\
\text { 'because occasionally we can get Tesco's slots' (P9; 18-24; Female; White; GCSEs or equivalent; Primary, 2) } \\
\text { 'The only thing I found really difficult [about self-isolation] was, I hated having to ask someone else to go and buy me food' (P23; 25 } \\
-49 ; \text { Female; White; British; Higher education or equivalent; Primary, 2) } \\
\text { 'I think then we'd struggle because apart from anything, you can't get online deliveries and that at the moment' (P12; 25-49; } \\
\text { Female; White; A-Level or equivalent; Primary, 1) } \\
\text { 'I had to get a friend to do all my shopping for me. But thankfully I'd stocked up quite a lot of food, anyway, so it was all right. No, I } \\
\text { didn't panic buy, I always have loads of food in anyway' (P23; 25-49; Female; White; British; Higher education or equivalent; } \\
\text { Primary, 2) }\end{array}$ \\
\hline Limited space & $\begin{array}{l}\text { 'We live in a three-bedroom bungalow ... it would be virtually impossible. Especially if more than one person had it, which it's likely } \\
\text { to happen, in a house of six people' (P8; 25-49; Female; White; Higher education or equivalent; Secondary, 4) } \\
\text { 'It would be a challenge if [self-isolation] had happened. It's a relatively small house, it's got three bedrooms' (P21; 50-65; Male; } \\
\text { White; Higher education or equivalent; Secondary, } 3 \text { ) }\end{array}$ \\
\hline
\end{tabular}

BAME, Black, Asian and Minority Ethnic; GCSE, General Certificate of Secondary Education.

children. In our interview schedule, we asked parents whether their child had had 'coronavirus or coronavirus symptoms, either a high temperature or new continuous cough?'. We also asked a set of questions about whether the parent or child would find it difficult to self-isolate and how they would cope with self-isolation.

Results were analysed using an inductive approach to thematic analysis. ${ }^{7}$ We found four themes relating to symptom attribution and four themes relating to self-isolation. These are described in Table 1 with supporting quotes.

In terms of symptom attribution, parents appeared to go through a process of finding the most likely reason for the experience of symptoms, discounting COVID-19 if a more likely explanation was apparent ('normalising symptoms'). Symptoms were often normalised in day-to-day terms that reduced the perceived risk and thus the intention to isolate. Particularly if symptoms were transitory or mild, this reduced worry and increased the likelihood of attribution to a non-COVID explanation. Conversely some participants expressed a view that 'any symptoms' should be treated as if they were COVID-19 related ('err on the side of caution'). Given the context of the pandemic, they would isolate if they or a household member experienced either a cough or fever, to be on the safe side.

Some parents indicated that they would be more likely to attribute high temperature than a cough to COVID-19 ('experience of temperature'). While a cough could be put down to a sore throat or common cold, parents appeared more cautious about a temperature.

There was a sense among some participants that unexpected or unusual symptoms would be a particular cause for concern ('symptoms not normal for us'). Annual or seasonal experience of symptoms (e.g. hay fever) reduced parental concerns about whether symptoms were due to COVID-19 or not, as well as the experience of symptoms 'normal' to their household. Conversely, symptoms that were 'weird' or unusual for the person were 'a key indicator if something is wrong.'

Parents had varied beliefs about their ability to isolate from others in the home and particularly the difficulty of isolating from children ('difficult to prevent contact with children'). Across interviews, parents expressed that this was related to their child's age and their understanding of the virus, i.e. younger children do not understand boundaries or reasons why they cannot be close to their parents. A common, fatalistic sentiment was that if one member of their household presented symptoms of COVID-19, then the whole household would catch it. Single parents relied on novel sources of care when they themselves became ill - for example, increased dependence on online resources, such as gaming and YouTube videos. When having to isolate due to symptoms, one parent stated they sent their child to their grandparent's house so that they could get sufficient rest to recover.

At the time of data collection, people could only leave home for very limited reasons (for example, shopping as infrequently as possible and a daily walk or exercise). Some parents noted that it would be easy to isolate the entire household as it would be no different to how they were already living during lockdown ('isolation would be no different to lockdown life').

Access to additional help or available resources was identified as impacting parents' ability to isolate. It would appear that ability to self-isolate was facilitated by connectedness to other members of the community and access to local shops ('ability to get food and supplies'); lack of this may make parents believe that they are not able to isolate. Some parents identified that the size of the home would be an additional challenge during self-isolation ('limited space').

Although rules and context have changed because the data were collected early in the pandemic, the findings highlight several key areas worthy of further exploration and quantification. We believe that there are two main implications.

First, parents' perceptions of whether a given symptom is a possible indicator of COVID-19 do not match the official guidance. Symptoms are often not attributed to COVID-19 unless a temperature is present. Data from a national UK study suggest that among those younger than 18 years, $48 \%$ of those who tested positive for COVID-19 reported having a temperature in the first 7 days of the illness. ${ }^{8}$ Because parents who identified 'not normal' symptoms or a temperature in themselves or their child were more inclined to attribute them to COVID-19, communications may benefit from highlighting that the presence of even one of the identified symptoms - i.e. a cough alone - necessitates self-isolation or request of a test, even if that symptom is mild. ${ }^{6}$

Second, isolation is seen as difficult by many parents. Given the guidance at the time of interviewing, parents ideally should have isolated from their children to the best of their ability. Many parents identified that this would be difficult or 'impossible'. Our findings also suggest that larger families and those living in smaller homes may find it particularly difficult to isolate. Indeed, research suggests that households with dependent children are less likely to adhere to self-isolation than those without children. ${ }^{9}$ Those who perceived household isolation to be easier were those who said it would be no different to their life in lockdown and would isolate even if they were not sure their or their child's symptoms were due to COVID19. Parents who reported that they could rely on someone for help with food shopping were more likely to think they could isolate. This is in line with previous research. ${ }^{10}$ We suggest that 
aiding families with grocery shopping may be a practical route to facilitating adherence to isolation. Although it might be impractical for supermarkets to do this, grassroots organisations played a key role in the first wave and could mobilise again in the second wave.

\section{Author statements}

\section{Ethical approval}

All participants received information sheets. Informed consent was provided electronically, before the interviews. The research was approved by the Psychiatry, Nursing and Midwifery Research Ethics Subcommittee at King's College London (ref MRSP-19/20-18349).

\section{Funding}

This study was funded by the Economic and Social Research Council [grant number ES/P000703/1] and the National Institute for Health Research Health Protection Research Unit (NIHR HPRU) [grant number NIHR200890] in Emergency Preparedness and Response, a partnership between Public Health England, King's College London and the University of East Anglia. The views expressed are those of the authors and not necessarily those of the NIHR, Public Health England or the Department of Health and Social Care.

\section{Competing interests}

G.J.R. and L.E.S. are participants in meetings of UK Scientific Advisory Group for Emergencies and its subgroups.

\section{Author contributions}

L.W. designed the interview schedule. A.H. and L.W. conducted interviews. A.H. wrote the first draft of the article with input from L.W., L.E.S., and G.J.R.
Role of the funding source

The sponsor and funder of the study had no role in study design, analysis, interpretation, or writing of the report. The corresponding author had full access to all the data in the study and had final responsibility for the decision to submit for publication.

\section{References}

1. HM Government. Health protection in schools and other childcare facilities London: HM Government; 2020 [updated 27 March 2019; cited 2020 1st October].

2. Adams R, Stewart H. UK schools to be closed indefinitely and exams cancelled. The Guardian; 2020 [cited 2020 1st October]. Available from: https://www. theguardian.com/world/2020/mar/18/coronavirus-uk-schools-to-be-closedindefinitely-and-exams-cancelled.

3. Otte im Kampe E, Lehfeld A-S, Buda S, Buchholz U, Haas W. Surveillance of COVID-19 school outbreaks, Germany, March to August 2020. Euro Surveill 2020;25(38):2001645.

4. Smith LE, Weinman J, Yiend J, Rubin J. Psychosocial factors affecting parental report of symptoms in children: a systematic review. Psychosom Med 2020;82(2):187-96.

5. Roberts M. Coronavirus symptoms: UK adds loss of smell and taste to list. BBC News; 2020.

6. NHS UK. When to self-isolate and what to do. 2020 [cited 2020 1st October]. Available from: https://www.nhs.uk/conditions/coronavirus-covid-19/selfisolation-and-treatment/when-to-self-isolate-and-what-to-do \#: : text=When\%20to\%20get\%20a\%20test,of\%20smell\%20or\%20taste.

7. Braun V, Clarke V. Using thematic analysis in psychology. Qual Res Psychol 2006;3(2):77-101.

8. Covid Symptom Study. Surprising research findings on the early symptoms of COVID-19. 2020 [cited 2020 1st October]. Available from: https://covid.joinzoe. com/post/early-covid-signs.

9. Smith LE, Potts HWW, Amlot R, Fear NT, Michie S, Rubin J. Adherence to the test, trace and isolate system: results from a time series of 21 nationally representative surveys in the UK (the COVID-19 Rapid Survey of Adherence to Interventions and Responses [CORSAIR] study). medRxiv 2020. https://doi.org/ 10.1101/2020.09.15.20191957.

10. Smith LE, Amlôt R, Lambert H, Oliver I, Robin C, Yardley L, et al. Factors associated with adherence to self-isolation and lockdown measures in the UK: a cross-sectional survey. Public Health 2020;187:41-52. 